\title{
Study on Three-Dimensional Analysis Method of Radiation Dose of GEO Satellite
}

\author{
Chunsheng $X u^{*}$, Yang Zhang, Hui Liu, Yong Yang and Yongquan Xia \\ China Academy of Space Technology, Beijing, China
}

\begin{abstract}
GEO satellite in orbit is greatly affected by the total radiation dose of space environment. In order to meet the requirements of satellite reliability design, radiation dose analysis is an important work in the process of satellite design. Previous radiation dose analysis is based on one-dimensional dose-depth analysis, or three-dimensional analysis, but over-simplified 3-D model may cause the analysis results deviated different from the reality. This paper presents a three-dimensional radiation dose analysis method based on the actual 3-D model of satellite. This method could analyze radiation dose with actual models, which could be automatically simplified according to needs. It could automatically extract the attribute information in the model and calculate the radiation dose value in each subspace
\end{abstract}

\section{Introduction}

The geostationary orbit communication satellite(GEO satellite) operates in the space where there are charged particles from the radiation environment of the earth radiation belt, solar cosmic ray and galactic cosmic ray plasma. These charged particles interact with the electronic components and materials used by the satellite, which can produce ionization total dose effect, displacement damage effect, single event effect, surface charge/discharge effect, inner charged effect and so on. Radiation effects have an impact on the performance of function of satellite and their subsystems, equipment, components and materials, which can even cause complete loss of functions in severe cases[1-3]. On one hand, the results of radiation dose analysis at specified points in the satellite is the basis for overall designers to optimize the layout of equipments, on the other hand, it is also the basis of further analysis by the equipment designer. In the aspect of radiation dose analysis, there are two main forms: one-dimensional analysis base on solid sphere shielding model and three-dimensional analysis based on whole satellite overall layout shielding model $[4,5]$. The three-dimensional analysis reflects the most real radiation shielding situation of satellite and *Corresponding author: xcstju@126.com reflects the anisotropic characteristics of satellite radiation shielding. In three-dimensional analysis area, sector analysis tool(SSAT)[6] is a shielding and radiation dose assessment tool which based on Monte Carlo software Geant 4 and widely used by ESA. But SSAT has to transform the abnormal complex structure models of satellite into geometric description model(GDML) before recognizing them. The workload of model transformation is large, and some information will be lost during the process too. In order to simplify the calculation, the models have to be simplified, which make the analysis models and analysis consequences different from the real state.

On the basis of one-dimensional analysis data and the secondary development function CATIA software, a three-dimensional radiation dose analysis method based on real satellite 3-D model is designed in this paper. This method is completely based on satellite model and can automatically extract model parameters and simplify the model according to needs. It can also give the total dose of checked points and the dose values in various directions.

\section{3-D analysis of radiation dose}




\subsection{Dose-depth curve modeling}

The basis of three-dimensional analysis of radiation dose is one-dimensional dose-depth curve, which comes from commercial software. The particle environment in the capture zone of geosynchronous orbit varies with the location of the satellite. The radiation dose in the 15-year typical orbit is constructed as shown in Table 1. Only the total dose of equivalent aluminum thickness from $0.015 \mathrm{~mm}$ to $0.5 \mathrm{~mm}$ are listed in the table. In practical application, the total dose data of equivalent aluminum thickness up to $20 \mathrm{~mm}$ are generally given. The total dose in one direction is calculated according to the shielding surface density of this direction, so the data in Table 1 need to be interpolated. According to engineering practice, simple polynomial interpolation is adopted. As seen in Table 1, "The total dose" changes relatively dramatically relative to "the shielding surface density", which is not suitable to use a single interpolation formula, and piecewise interpolation can be carried out here. It has been proved that the application of three-section interpolation is appropriate. The results are shown in Figure 1.

Table 1. In orbit depth-dose data for satellite

\begin{tabular}{cccccc}
\hline $\begin{array}{c}\text { equivalent aluminum } \\
\text { thickness }(\mathrm{mm})\end{array}$ & $\begin{array}{c}\text { Shielding } \\
\text { surface dense } \\
\left(\mathrm{g} / \mathrm{cm}^{2}\right)\end{array}$ & $\begin{array}{c}\text { Capture electron dose and } \\
\text { bremsstrahlung radiation dose } \\
{[\mathrm{rad}(\mathrm{Si})]}\end{array}$ & $\begin{array}{c}\text { capture proton } \\
\text { dose } \\
{[\mathrm{rad}(\mathrm{Si})]}\end{array}$ & $\begin{array}{c}\text { Solar flares } \\
\text { proton dose } \\
{[\mathrm{rad}(\mathrm{Si})]}\end{array}$ & $\begin{array}{c}\text { Total } \\
\text { dose } \\
{[\mathrm{rad}(\mathrm{Si})]}\end{array}$ \\
\hline 0.015 & 0.004 & $1.44 \mathrm{E}+09$ & $3.22 \mathrm{E}+03$ & $3.44 \mathrm{E}+05$ & $1.44 \mathrm{E}+09$ \\
0.1 & 0.027 & $5.20 \mathrm{E}+08$ & $0.00 \mathrm{E}+00$ & $1.15 \mathrm{E}+05$ & $5.20 \mathrm{E}+08$ \\
0.2 & 0.054 & $2.67 \mathrm{E}+08$ & $0.00 \mathrm{E}+00$ & $6.80 \mathrm{E}+04$ & $2.67 \mathrm{E}+08$ \\
0.3 & 0.081 & $1.58 \mathrm{E}+08$ & $0.00 \mathrm{E}+00$ & $4.88 \mathrm{E}+04$ & $1.58 \mathrm{E}+08$ \\
0.4 & 0.108 & $1.05 \mathrm{E}+08$ & $0.00 \mathrm{E}+00$ & $3.78 \mathrm{E}+04$ & $1.06 \mathrm{E}+08$ \\
0.5 & 0.135 & $7.47 \mathrm{E}+07$ & $0.00 \mathrm{E}+00$ & $3.06 \mathrm{E}+04$ & $7.48 \mathrm{E}+07$ \\
\hline
\end{tabular}

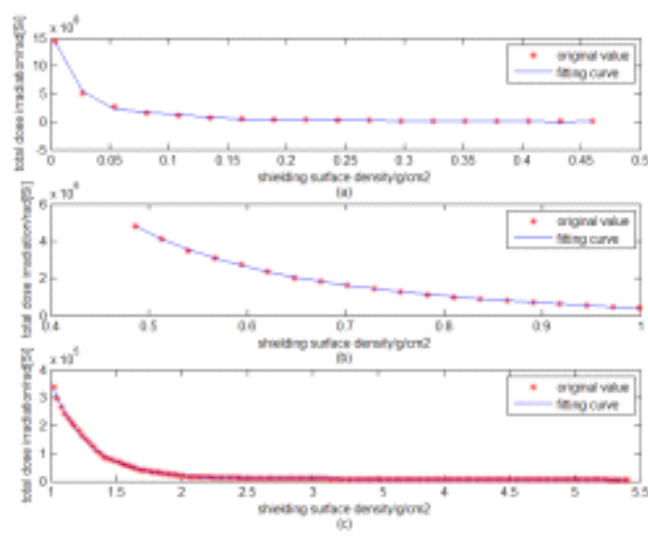

Fig. 1. Dose-depth curve interpolation

\subsection{3-D radiation dose analysis process}

After obtaining the one-dimensional dose-depth curve, the total radiation dose can be analyzed based on the 3-D model of satellite. The main ideas are as follows: Firstly, the 3-D model of satellite is processed. Secondly, the relevant information of each 3-D model of equipment is extracted. If the simplified model is selected, the model can be simplified according to the information of the extracted from 3-D model. Then, fixed dose points are divided into omni directional space to calculate the shielding surface density and equivalent aluminum thickness in omni directional space. Finally, the total radiation dose of the designated checked points is calculated, and the shielding surface density of each subspace is displayed in three dimensions.

According to the above idea, the total analysis process is shown in Figure.2. 


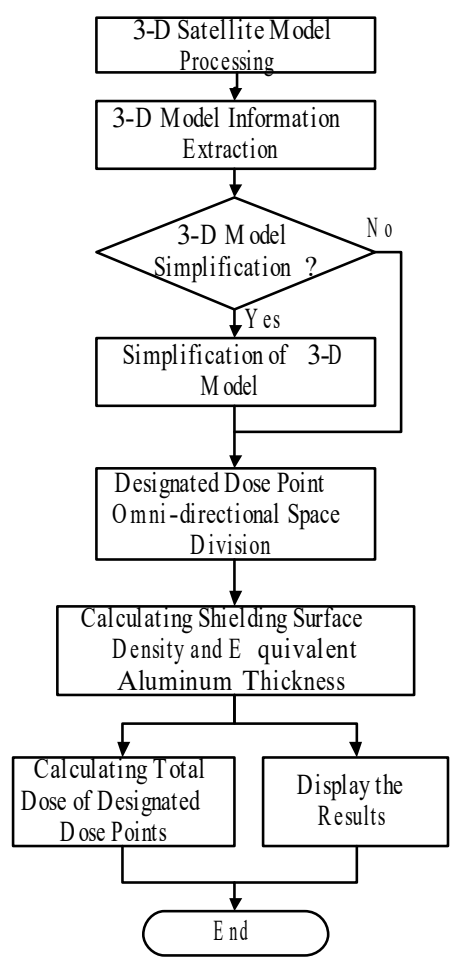

Fig. 2. Three-dimensional analy sis flow of radiation dose

\subsection{Processing and information extraction of satellite model}

\subsubsection{Model processing of satellite's equipments}

Firstly, it is necessary to use the secondary development function of the three-dimensional design software to endow the models of equipments with their parameters information, such as mass, center of gravity(CoG), moment of inertia. These parameters are input for subsequent model simplification and calculation of shielding surface density.

In addition, if the models have no parameters listed above, the software can traverse the whole models by using the product matching tables, with which corresponding models can be correlated by equipment name, so the corresponding parameters of each equipment can be automatically extracted and stored in a database.

\subsubsection{3-D Model Information Extraction of Equipment}

For the equipment with parameters information, the program can read its information directly, and the secondary development can obtain the volume of each
3-D model, based on the density of the corresponding model according to the real quality. If the equipment does not have the above information, the quality parameters stored in the database can be invoked. Then the volume and moment of inertia of each model can be obtained by using the secondary development program, and the density of the corresponding equipment can be obtained by using the mass and volume of the model.

\subsubsection{Simplification of 3-D Model of Equipment}

In order to improve the speed of dose analysis, the equipment model can be simplified to a certain extent, such as removing the electrical connector, socket ears, structural protrusions and other parts, and simplifying the model to simple geometries such as cuboids or cylinders.

The length, width and height of the model can be calculated according to the $\mathrm{CoG}$ and moment of inertia extracted from the equipment information(see Formula 1), so that the simplified model can be quickly established and the automatic assembly of the simplified model can be realized.

$X=\sqrt{\frac{6\left(I_{y y}+I_{z z}-I_{x x}\right)}{m}}, Y=\sqrt{\frac{6\left(I_{x x}+I_{z z}-I_{y y}\right)}{m}}, Z=\sqrt{\frac{6\left(I_{x x}+I_{y y}-I_{z z}\right)}{m}}$

In formula $1, X 、 Y 、 Z$ are respectively the length, width, and height of the simplified model. Ixx、Iyy、Izz are the principal inertia of the model. $m$ is the mass of the model.

\subsubsection{Space Division of Designated Dose Points}

The CoG or geometric center of the analytic equipment model is usually selected for the setting of dose points.

The CoG position can be obtained using secondary development, and also could set the dose point coordinates manually.

Taking the dose point as the center of the sphere, the omni directional space is used with a cylindrical body with a certain radius(the smaller the radius of the cylindrical body, the more accurate the calculation is).In this article, the diameter of the cylinder is taken as $1 \mathrm{~mm}$ to divide it evenly. The denser the partition space, the closer the calculation will get to reality, but too dense partitioning makes the computing time prolong correspondingly. After comprehensive consideration, 
omni directional space is divided into 10,000 subspaces.

\subsubsection{Radiation Dose Calculation}

In each subspace, the volume of the model penetrating the cylindrical body representing the space and all the equipments on the satellite is calculated. Then, according to the density of the model penetrating and the cross-sectional area of the cylindrical body, the shielding surface density (i.e. the density multiplied by the cross-sectional area) of the subspace is calculated. After obtaining the shielding surface density, the equivalent aluminum thickness of the subspace can be obtained according to Formula: $\mathrm{H}=\rho / 2.7 \mathrm{E}(-3), \rho$ is the density of calculated shielding surface, and is the density of penetrated 3-D model multiplied by the penetration length, and its unit is $\mathrm{g} / \mathrm{mm} 3 . \mathrm{H}$ is the calculated equivalent thickness of aluminum, and its unit is $\mathrm{mm}$.

After obtaining the equivalent aluminum thickness of each subspace, the radiation dose of the subspace can be obtained according to the one-dimensional depth-dose curve. The radiation dose of each subspace is superposed, and the superposed value is averaged according to the number of subspaces, then the total radiation dose at the dose point is obtained.

\subsubsection{Post-processing Design}

The radiation dose values of each subspace are displayed by three-dimensional mesh graph, which makes it easy for designers to intuitively judge the direction of relatively weak radiation protection and take necessary measures.

\section{Application Verification}

Constructing a satellite model is shown in Figure. 3.

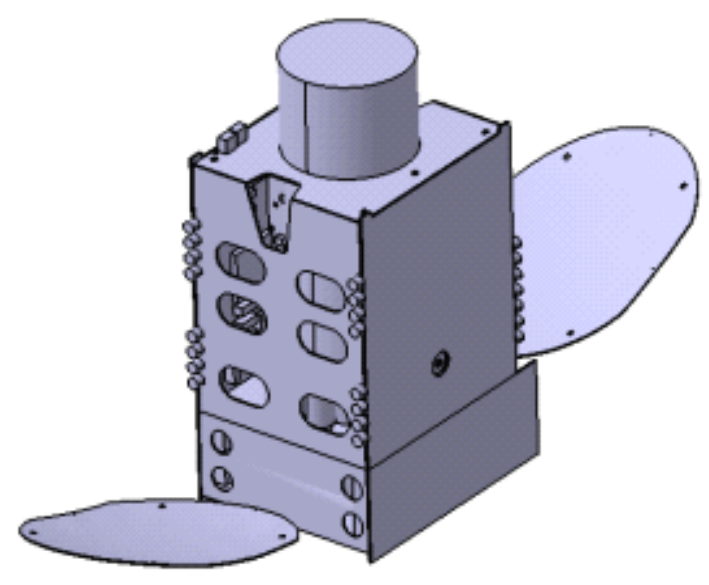

Fig. 3. Constructed satellite model

After that, dose points are set and omni directional space is divided. When dividing omni directional space, the method of constructing small diameter cylinder is adopted. In order to simplify calculation, more than 400 cylinders are constructed, as shown in Figure.4.

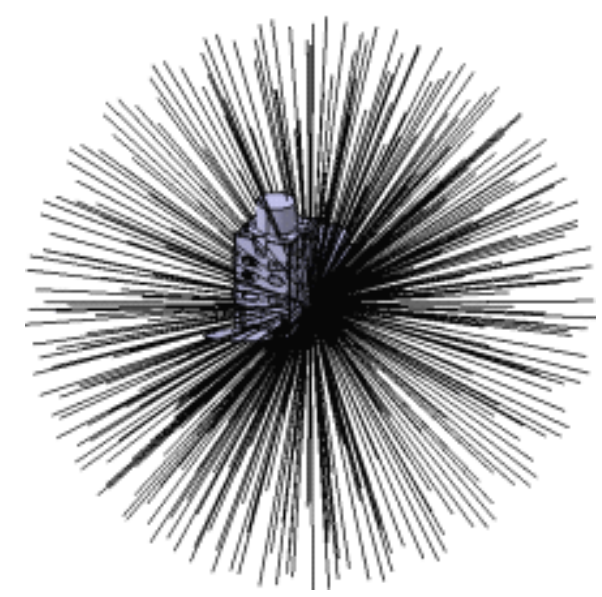

Fig. 4. Models after partitioning space

Next, for each cylinder used to divide the space, the interference between the cylinder and the 3-D model of the equipment on the satellite is calculated, and the shielding surface density and the equivalent aluminum thickness of the subspace represented by the cylinder are calculated according to the one-dimensional depth-dose curve. The calculation results are shown in Table 2. In Table 2, only the density of shielding surface in the first five spaces is given. "Quantity " refers to the number of interference between the cylinder representing each subspace and the equipment model on the satellite in Figure.4, "IfV(Inference Volume)" refers to the volume of the equipment model with which each cylinder penetrates, "SD(Surface Density)" refers to the 
Table 2 Density values of shielding surfaces in each

\begin{tabular}{|c|c|c|c|c|c|c|c|c|}
\hline Num & Subspace & Quantity & $\mathrm{SD}\left(\mathrm{g} / \mathrm{cm}^{2}\right)$ & IfV $1\left(\mathrm{~mm}^{3}\right)$ & IfV $2\left(\mathrm{~mm}^{3}\right)$ & IfV $3\left(\mathrm{~mm}^{3}\right)$ & IfV $4\left(\mathrm{~mm}^{3}\right)$ & IfV $5\left(\mathrm{~mm}^{3}\right)$ \\
\hline 1 & Subspace 1 & 4 & 16.73 & 210.37 & 16.56 & 20.35 & 788.57 & \\
\hline 2 & Subspace 2 & 3 & 9.29 & 16.66 & 60.27 & 20.47 & & \\
\hline 3 & Subspace 3 & 4 & 26.59 & 16.83 & 158.77 & 58.11 & 20.68 & \\
\hline 4 & Subspace 4 & 5 & 33.17 & 17.07 & 28.90 & 184.11 & 58.95 & 20.98 \\
\hline 5 & Subspace 5 & 5 & 35.83 & 17.40 & 52.84 & 166.11 & 22.50 & 64.57 \\
\hline
\end{tabular}

equivalent surface density generated by the protection of other equipments in this subspace.subspace

Finally, the shielding surface density and equivalent aluminum thickness of each subspace can be obtained, as shown in Figure.5. Then, the total radiation dose of the designated dose point in the whole space is obtained.

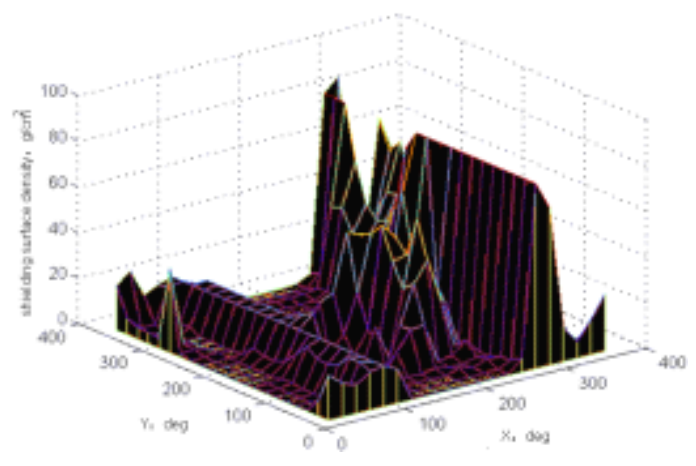

Fig. 5. Three-dimensional representation of shielding surface density in each subspace

\section{Concluding Remark}

In order to solve the shortage of one-dimensional analysis of radiation dose, a three-dimensional analysis method of radiation dose closely combined with satellite model is presented to guide the design of total dose radiation protection of satellite. Using this method, we can calculate the shielding surface density of any subspace with any specified dose on the satellite, calculate the total radiation dose of any designated dose point on the satellite, and display the shielding surface density of each subspace in the omni directional space in three dimensions, so that the designer can intuitively understand which subspace needs to take further shielding measures.

\section{References}

1. Yuxiong Xue ,Zhou Cao, Shiyu Yang, et al. The analysis of typical GEO space radiation environment based on the space radiation 5.0 platform. J. Spacecraft environment engineering, 24,5(2007):291-295

2. Xu Sen, Ma Jun, Liu jia. Space environment analysis of small satellite based on MATLAB. C.The collection of the theses on the development of modern small satellite technology in China(2015)

3. Luo Yinhong, Zhang Fengqi, Guo Hongxia, et al. Experimental study on heavy-ion single event effect on nanometer DDR SRAM. J. High Power Laser and Particle Beams, 25,6(2013)

4. Zhengbo Cai, Analysis of space radiation dose of bisatellite exploration satellite. C. Tenth outstanding academic meeting of the committee on space exploration of the Chinese society of space sciences(2003)

5. Qing Sun, Analysis of Mass Shielding Property of Satellite. D.Tianjin University(2008)

6. QinetiQ, Inc. Space information systems. sector shielding analysis tool based on geant $4[\mathrm{~EB} / \mathrm{O}$ L]. http://www.space.qinetiq.com/geant $4 /$ s sat.html (2001). 